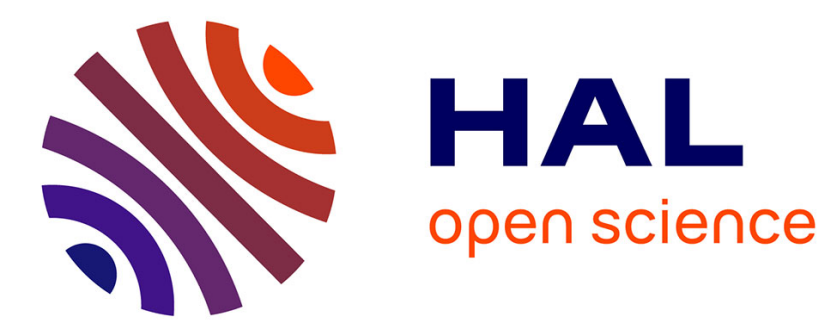

\title{
Asymptotic Modeling of piezoelectric plates with electric field gradient
}

\author{
Thibaut Weller, Christian Licht
}

\section{To cite this version:}

Thibaut Weller, Christian Licht. Asymptotic Modeling of piezoelectric plates with electric field gradient. Comptes Rendus Mécanique, 2012, 340, pp.405-410. 10.1016/j.crme.2012.02.020 . hal-00742878

\section{HAL Id: hal-00742878 \\ https://hal.science/hal-00742878}

Submitted on 17 Oct 2012

HAL is a multi-disciplinary open access archive for the deposit and dissemination of scientific research documents, whether they are published or not. The documents may come from teaching and research institutions in France or abroad, or from public or private research centers.
L'archive ouverte pluridisciplinaire HAL, est destinée au dépôt et à la diffusion de documents scientifiques de niveau recherche, publiés ou non, émanant des établissements d'enseignement et de recherche français ou étrangers, des laboratoires publics ou privés. 


\title{
Asymptotic modeling of piezoelectric plates with electric field gradient
}

\author{
Thibaut Weller, Christian Licht \\ Laboratoire de Mécanique et Génie Civil, UMR 5508 CNRS - UMII, Université Montpellier II, c.c. 048, \\ Place Eugène Bataillon, 34095 Montpellier cedex 5, France \\ Received *****; accepted after revision +++++ \\ Presented by
}

\begin{abstract}
We extend our modeling of smart structures to second order piezoelectricity. We show that three different models have to be taken into account, which broadens the scope of the sensors and actuators field. Second order piezoelectricity being compatible with isotropy, we also propose a systematic study of the impact of crystalline symmetries on our models. To cite this article: T. Weller, C. Licht, C. R. Mecanique xxx (aaaa).

\section{Résumé}

Modélisation asymptotique de plaques piézoélectriques avec gradient du champ électrique Nous étendons à la piézoélectricité avec gradient du champ électrique notre étude des couplages électromécaniques dans les structures minces. Nous montrons en particulier que trois modèles distincts apparaissent à la limite, ce qui enrichit substantiellement la description du comportement des capteurs et actionneurs en fonction du chargement électrique qui leur est imposé. La piézoélectricité du second gradient étant compatible avec l'isotropie, nous proposons également une étude systématique de l'influence des symétries cristallines sur les modèles obtenus. Pour citer cet article: T. Weller, C. Licht, C. R. Mecanique xxx (aaaa).
\end{abstract}

Key words: Asymptotic modeling, piezoelectric plates, electric field gradient.

Mots-clés : Analyse asymptotique, plaques piézoélectriques, gradient du champ électrique.

Email addresses: thibaut.weller@univ-montp2.fr (Thibaut Weller), clicht@univ-montp2.fr (Christian Licht). 


\section{Introduction}

In the 1960's the study of unexplained aspects of piezoelectricity led Mindlin [1] to extend the classical Voigt theory [2] in Toupin's formulation [3] by assuming that the stored energy function not only depends on the strain tensor and polarization vector but also on the polarization gradient tensor. What motivated Mindlin to study the effects of the polarization gradient was the capacitance of a very thin dielectric film. Experiments showed that the capacitance of a very thin film is systematically smaller than the classical prediction. Moreover, performing experimental tests, Mead [4] showed that piezoelectric effects can also appear in centrosymmetric crystals, which is in contradiction with classical Voigt theory. And, indeed, the Mindlin's theory of elastic dielectrics with polarization gradient accomodates the observed and experimentally measured phenomena, such as electromechanical interactions in centrosymmetric materials, capacitance of thin dielectric films, surface energy of polarization, deformation and optical activity in quartz (see for example [5], [6] and references quoted therein). In this paper we choose to adopt an alternative to the Mindlin formulation by introducing the electric field gradient, as in [7] for example. Because such gradient theories can describe size effects that are important in small-scale problems, it seems unavoidable to use them to deepen our understanding of smart structures, the wide majority of them being thin. In this paper we perform a mathematical modeling of second order piezoelectric plate by regarding its thickness as a small parameter denoted by $\varepsilon$. Then, we study the behavior of the solution of the electromechanical problem as $\varepsilon$ tends to 0 . We show that depending on the type of electric loading, three different models indexed by $p$ appear at the limit. This result extends our previous study in [8] and shows that gradient theory broadens the understanding of sensors and actuators. When $p=2$ and $p=3$ we are able to express the constitutive laws as a Schur complement of the second order piezoelectric tensor (see (2) and (11)). It is important to emphasize that this expression is valid for any symmetry class, which means that we do not make any simplifying assumptions dealing with the crystal symmetry of the material constituting the plate. When $p=1$, we are not able to explicitly derive the constitutive law of the limit model. Therefore, as in the case of first order piezoelectric rods treated in [9], it seems very likely to us that non-local terms appear in this delicate situation. Finally, we study the influence of the crystal symmetries on our models for $p=2,3$ and show that even for second order piezoelectricity, an electromechanical switch-off may appear in the structure if the plate is designed with specific materials.

\section{Setting the problem}

We will denote displacement fields by the letters $u, v$ and $w$ while the electric potentials will be denoted by $\varphi, \psi$ and $\phi$. Depending on the nature of our formulation, these letters may be indexed by $\varepsilon$ which stands for the thickness of the plate, regarded as a parameter. Classically, the tensor of small strains is written $e(u) \in S^{3}$ where $S^{N}$ indicates the set of all $N \times N$ real and symmetric matrices. Used as indexes, letters $i$ and $j$ take their values in $\{1,2,3\}$ while $\alpha$ and $\beta$ take their values in $\{1,2\}$. We recall that $2 e_{i j}(u)=\partial_{i} u+\partial_{j} u$ where the symbol $\partial_{i}$ refers to the partial derivative with respect to the $i$ th coordinate. The gradient of an electric potential $\varphi$ will be denoted by $\nabla \varphi \in \mathbb{R}^{3}$ and its bigradient by $\nabla^{2} \varphi \in S^{3}$ where $\nabla_{i j}^{2} \varphi=\partial_{i j}^{2} \varphi=\partial_{i} \partial_{j} \varphi$. Given an electromechanical state $(u, \varphi)$ we therefore have $\left(e(u), \nabla \varphi, \nabla^{2} \varphi\right) \in \mathcal{H}$, with

$$
\mathcal{H}=S^{3} \times \mathbb{R}^{3} \times S^{3} .
$$

Often, an element of $\mathcal{H}$ will be represented by a triplet $(e, g, G)$. For the sake of simplicity, the classical symbol - will stand for the scalar product in $\mathcal{H}, S^{3}$ and $\mathbb{R}^{3}$. The set of all linear mappings from a space $V$ into a space $W$ is denoted by $\mathcal{L}(V, W)$ and, if $V=W$, we simply write $\mathcal{L}(V)$. In the sequel, for all 
domain $D$ of $\mathbb{R}^{N}, H_{\Gamma}^{1}(D)$ refers to the subset of the Sobolev space $H^{1}(D)$ whose elements vanish on $\Gamma$, included in the boundary $\partial D$ of $D$, except $H_{m}^{1}(D)$ which is the set of the elements of $H^{1}(D)$ with zero average on $D$.

The reference configuration of a linearly piezoelectric thin plate is the closure in $\mathbb{R}^{3}$ of the set $\Omega^{\varepsilon}=$ $\omega \times(-\varepsilon, \varepsilon)$ whose outward unit normal is $n^{\varepsilon}$. Here, $\varepsilon$ is a small positive number and $\omega$ a bounded domain of $\mathbb{R}^{2}$ with a Lipschitz boundary $\partial \omega$. The lateral part of the plate $\partial \omega \times(-\varepsilon, \varepsilon)$ is denoted $\Gamma_{\text {lat }}^{\varepsilon}$, while $\Gamma_{ \pm}^{\varepsilon}=\omega \times\{ \pm \varepsilon\}$ refers to the upper or lower face, respectively. Let $\left(\Gamma_{m D}^{\varepsilon}, \Gamma_{m N}^{\varepsilon}\right),\left(\Gamma_{e D, i}^{\varepsilon}, \Gamma_{e N, i}^{\varepsilon}\right)_{i=1,2}$ three suitable partitions of $\partial \Omega^{\varepsilon}$ with $\Gamma_{m D}^{\varepsilon}$ and $\Gamma_{e D, i}^{\varepsilon}$ of strictly positive surface measures. The plate is, on one hand, clamped along $\Gamma_{m D}^{\varepsilon}$ and the electric potential $\varphi^{\varepsilon}$ satisfies $\varphi^{\varepsilon}=\varphi_{0}^{\varepsilon}$ on $\Gamma_{e D, 1}^{\varepsilon}$ and $\partial_{n} \varphi^{\varepsilon}=\partial_{n} \varphi_{0}^{\varepsilon}$ on $\Gamma_{e D, 2}^{\varepsilon}$, where the symbol $\partial_{n}$ refers to the normal derivative along the boundary of $\Omega^{\varepsilon}$ and $\varphi_{0}^{\varepsilon}$ is a smooth enough given field defined in $\Omega^{\varepsilon}$. On the other hand, the plate is subjected to body forces $f^{\varepsilon}$ and electric loading $q^{\varepsilon}$ in $\Omega^{\varepsilon}$. Actually, $q^{\varepsilon}$ vanishes, the material being an insulator, anyway the following analysis stands with $q^{\varepsilon}$ different from 0 . Moreover, the plate is subjected to surface forces $F^{\varepsilon}$ and electric loading $q_{s}^{\varepsilon}$ on $\Gamma_{m N}^{\varepsilon}$ and $\Gamma_{e N, 1}^{\varepsilon}$ respectively. It is also necessary to define 'body' and 'surface' electric dipoles densities, respectively denoted by $d^{\varepsilon}, d_{s}^{\varepsilon}$ and defined in $\Omega^{\varepsilon}$ and on $\Gamma_{e N, 2}^{\varepsilon}$. Finally, we assume that $\Gamma_{m D}^{\varepsilon}=\gamma_{0} \times(-\varepsilon, \varepsilon)$, with $\gamma_{0} \subset \partial \omega$.

We now define the operator

$$
M^{\varepsilon}=\left(\begin{array}{ccc}
a^{\varepsilon} & -b^{\varepsilon} & -\alpha^{\varepsilon} \\
b^{\varepsilon^{T}} & c^{\varepsilon} & \beta^{\varepsilon} \\
\alpha^{\varepsilon^{T}} & \beta^{\varepsilon^{T}} & \gamma^{\varepsilon}
\end{array}\right)
$$

which describes the electromechanical coupling with electric field gradient effect. More precisely, $a^{\varepsilon}, b^{\varepsilon}$ and $c^{\varepsilon}$ are respectively the elastic, piezoelectric and dielectric tensors while $\alpha^{\varepsilon}, \beta^{\varepsilon}$ and $\gamma^{\varepsilon}$ describe the second order couplings. Recall that $\theta^{T}$ denotes the transpose of any tensor $\theta$. We have $\left(a^{\varepsilon}, b^{\varepsilon}, c^{\varepsilon}, \alpha^{\varepsilon}, \beta^{\varepsilon}, \gamma^{\varepsilon}\right) \in$ $\mathcal{L}\left(S^{3}\right) \times \mathcal{L}\left(\mathbb{R}^{3}, S^{3}\right) \times \mathcal{L}\left(\mathbb{R}^{3}\right) \times \mathcal{L}\left(S^{3}\right) \times \mathcal{L}\left(S^{3}, \mathbb{R}^{3}\right) \times \mathcal{L}\left(S^{3}\right)$, so that $M^{\varepsilon} \in \mathcal{L}(\mathcal{H})$

We are looking for the electromechanical state $\left(u^{\varepsilon}, \varphi^{\varepsilon}\right)$ living in the piezoelectric plate at equilibrium, where $u^{\varepsilon}$ denotes the displacement field. For this purpose, we first make the following regularity hypothesis on the exterior loading:

$$
\left(\mathbf{H}_{1}\right):\left\{\begin{array}{l}
\left(f^{\varepsilon}, q^{\varepsilon}, d^{\varepsilon}, F^{\varepsilon}, q_{s}^{\varepsilon}, d_{s}^{\varepsilon}\right) \in L^{2}\left(\Omega^{\varepsilon}\right)^{3} \times L^{2}\left(\Omega^{\varepsilon}\right) \times L^{2}\left(\Omega^{\varepsilon}\right)^{3} \times L^{2}\left(\Gamma_{m N}^{\varepsilon}\right)^{3} \times L^{2}\left(\Gamma_{e N, 1}^{\varepsilon}\right) \times L^{2}\left(\Gamma_{e N, 2}^{\varepsilon}\right), \\
\varphi_{0}^{\varepsilon} \in H^{2}\left(\Omega^{\varepsilon}\right),
\end{array}\right.
$$

and define

$$
H_{\Gamma_{e D}^{\varepsilon}}^{2}\left(\Omega^{\varepsilon}\right)=\left\{\psi \in H^{2}\left(\Omega^{\varepsilon}\right): \psi=0 \text { on } \Gamma_{e D, 1}^{\varepsilon}, \partial_{n} \psi=0 \text { on } \Gamma_{e D, 2}^{\varepsilon}\right\} .
$$

Now, on the space of electromechanical states

$$
V^{\varepsilon}=H_{\Gamma_{m D}^{\varepsilon}}^{1}\left(\Omega^{\varepsilon}\right)^{3} \times H_{\Gamma_{e D}^{\varepsilon}}^{2}\left(\Omega^{\varepsilon}\right)
$$

we define a bilinear form $m^{\varepsilon}$ :

$$
\left.m^{\varepsilon}(r, t)=m^{\varepsilon}((v, \psi),(w, \phi))=\int_{\Omega^{\varepsilon}} M^{\varepsilon}\left(e(v), \nabla \psi, \nabla^{2} \psi\right)\right) \cdot\left(e(w), \nabla \phi, \nabla^{2} \phi\right) d x^{\varepsilon},
$$

and a linear form $L^{\varepsilon}$ : 


$$
L^{\varepsilon}(r)=L^{\varepsilon}((v, \psi))=\int_{\Omega^{\varepsilon}}\left(f^{\varepsilon} \cdot v+q^{\varepsilon} \psi+d^{\varepsilon} \cdot \nabla \psi\right) d x^{\varepsilon}+\int_{\Gamma_{m N}^{\varepsilon}} F^{\varepsilon} \cdot v d s^{\varepsilon}+\int_{\Gamma_{e N, 1}^{\varepsilon}} q_{s}^{\varepsilon} \psi d s^{\varepsilon}+\int_{\Gamma_{e N, 2}^{\varepsilon}} d_{s}^{\varepsilon} \partial_{n} \psi d s^{\varepsilon}
$$

The electromechanical problem then takes the form

$$
\mathcal{P}\left(\Omega^{\varepsilon}\right) \quad: \quad \text { Find } s^{\varepsilon}=\left(u^{\varepsilon}, \varphi^{\varepsilon}\right) \in\left(0, \varphi_{0}^{\varepsilon}\right)+V^{\varepsilon} \text { such that } m^{\varepsilon}\left(s^{\varepsilon}, r\right)=L^{\varepsilon}(r), \quad \forall r \in V^{\varepsilon} .
$$

Thus, with the additional and realistic assumptions of boundedness of $a^{\varepsilon}, b^{\varepsilon}, c^{\varepsilon}, \alpha^{\varepsilon}, \beta^{\varepsilon}, \gamma^{\varepsilon}$ and of uniform ellipticity of $a^{\varepsilon}, c^{\varepsilon}$ and $\gamma^{\varepsilon}$ :

$$
\left(\mathbf{H}_{2}\right) \quad: \quad M^{\varepsilon} \in L^{\infty}\left(\Omega^{\varepsilon}, \mathcal{L}(\mathcal{H})\right), \exists \kappa^{\varepsilon}>0: M^{\varepsilon}\left(x^{\varepsilon}\right) h \cdot h \geq \kappa^{\varepsilon}|h|_{\mathcal{H}}^{2}, \forall h \in \mathcal{H} \text {, a.e. } x^{\varepsilon} \in \Omega^{\varepsilon},
$$

the Stampacchia's theorem (cf. [10]) implies the

Theorem 2.1 Under assumptions $\left(\boldsymbol{H}_{1}\right)-\left(\boldsymbol{H}_{2}\right)$, the problem $\mathcal{P}\left(\Omega^{\varepsilon}\right)$ has a unique solution.

To derive a simplified and accurate model, the true question is to study the behavior of $s^{\varepsilon}$ when $\varepsilon$, regarded as a parameter, tends to zero.

\section{The three different models}

Here we will show that three different limit behaviors, indexed by $p=1,2$ or 3 , appear according to both the type of electric boundary conditions and the magnitude of the electric external loading. In the sequel, any $h=(e, g, G) \in \mathcal{H}$ will be represented as $\left(\widehat{e}, e^{\perp}, e_{33}, \widehat{g}, g_{3}, \widehat{G}, G^{\perp}, G_{33}\right)$ where the symbol denotes both elements of $\mathcal{L}\left(\mathbb{R}^{3}, \mathbb{R}^{2}\right)$ and $\mathcal{L}\left(S^{3}, S^{2}\right)$ defined by $\widehat{\xi}=\left(\xi_{1}, \xi_{2}\right)$ if $\xi \in \mathbb{R}^{3},(\widehat{\xi})_{\alpha \beta}=\xi_{\alpha \beta}$ if $\xi \in S^{3}$ while ${ }^{\perp}$ stands for the element of $\mathcal{L}\left(S^{3}, \mathbb{R}^{2}\right)$ such that $\left(\xi^{\perp}\right)_{\alpha}=\xi_{\alpha 3}$. For the sake of simplicity, we straightforwardly extend to differential operators the definitions of ${ }^{\wedge}$ and ${ }^{\perp}$.

Classicaly (see [11]) we come down to a fixed open set $\Omega=\omega \times(-1,1)$ through the mapping $\pi^{\varepsilon}$ :

$$
x=\left(\widehat{x}, x_{3}\right) \in \bar{\Omega} \mapsto \pi^{\varepsilon} x=\left(\widehat{x}, \varepsilon x_{3}\right) \in \bar{\Omega}^{\varepsilon} .
$$

Also, we drop the index $\varepsilon$ for the images by $\left(\pi^{\varepsilon}\right)^{-1}$ of the geometric sets defined in the preceding section. To get physically meaningful results, we have to make various kinds of assumptions. They deal with

(i) the electromechanical coefficients:

$\left(\mathbf{H}_{3}\right): M^{\varepsilon}\left(\pi^{\varepsilon} x\right)=M(x)$ with $M \in L^{\infty}(\Omega, \mathcal{L}(\mathcal{H})), \exists \kappa>0: M(x) h \cdot h \geq \kappa|h|_{\mathcal{H}}^{2}, \forall h \in \mathcal{H}$, a.e. $x \in \Omega$,

(ii) the electromechanical loading:

$$
\left(\mathbf{H}_{4}\right):\left\{\begin{array}{l}
\exists\left(f, F, q, d, q_{s}, d_{s}\right) \in L^{2}(\Omega)^{3} \times L^{2}\left(\Gamma_{m N}\right)^{3} \times L^{2}(\Omega) \times L^{2}(\Omega)^{3} \times L^{2}\left(\Gamma_{e N, 1}\right) \times L^{2}\left(\Gamma_{e N, 2}\right) ; \\
\widehat{f}^{\varepsilon}\left(\pi^{\varepsilon} x\right)=\varepsilon \widehat{f}(x), f_{3}^{\varepsilon}\left(\pi^{\varepsilon} x\right)=\varepsilon^{2} f_{3}(x), q^{\varepsilon}\left(\pi^{\varepsilon} x\right)=\varepsilon^{2-p} q(x), \forall x \in \Omega, \\
\widehat{F}^{\varepsilon}\left(\pi^{\varepsilon} x\right)=\varepsilon^{2} \widehat{F}(x), F_{3}^{\varepsilon}\left(\pi^{\varepsilon} x\right)=\varepsilon^{3} F_{3}(x), \forall x \in \Gamma_{m N} \cap \Gamma_{ \pm}, \\
\widehat{F}^{\varepsilon}\left(\pi^{\varepsilon} x\right)=\varepsilon \widehat{F}(x), F_{3}^{\varepsilon}\left(\pi^{\varepsilon} x\right)=\varepsilon^{2} F_{3}(x), \forall x \in \Gamma_{m N} \cap \Gamma_{l a t} \\
\widehat{d}^{\varepsilon}\left(\pi^{\varepsilon} x\right)=\varepsilon^{2-p} \widehat{d}(x), d_{3}^{\varepsilon}\left(\pi^{\varepsilon} x\right)=\varepsilon^{3-p} d(x), \forall x \in \Omega \\
q_{s}^{\varepsilon}\left(\pi^{\varepsilon} x\right)=\varepsilon^{3-p} q_{s}(x), \forall x \in \Gamma_{e N, 1} \cap \Gamma_{ \pm}, q_{s}^{\varepsilon}\left(\pi^{\varepsilon} x\right)=\varepsilon^{2-p} q_{s}(x), \forall x \in \Gamma_{e N, 1} \cap \Gamma_{l a t} \\
\widehat{d}_{s}^{\varepsilon}\left(\pi^{\varepsilon} x\right)=\varepsilon^{3-p} \widehat{d}_{s}(x), d_{s_{3}}^{\varepsilon}\left(\pi^{\varepsilon} x\right)=\varepsilon^{4-p} d_{s_{3}}(x), \forall x \in \Gamma_{e N, 2} \cap \Gamma_{ \pm} \\
\widehat{d}_{s}^{\varepsilon}\left(\pi^{\varepsilon} x\right)=\varepsilon^{2-p} \widehat{d}_{s}(x), d_{s_{3}}^{\varepsilon}\left(\pi^{\varepsilon} x\right)=\varepsilon^{3-p} d_{s_{3}}(x), \forall x \in \Gamma_{e N, 2} \cap \Gamma_{l a t} \\
\varphi_{0}^{\varepsilon}\left(\pi^{\varepsilon} x\right)=\varepsilon^{p} \varphi_{0}(x), \forall x \in \Omega .
\end{array}\right.
$$


(iii) the boundedness of the "work of the exterior loading":

$$
\left(\mathbf{H}_{5}\right):\left\{\begin{aligned}
p=1: & \text { the extension of } \varphi_{0} \text { into } \Omega \text { does not depend on } x_{3} . \\
p=2: & \text { - the closure } \overline{\delta_{\alpha}} \text { of the projection of } \Gamma_{e D, \alpha} \text { on } \omega \text { coincides with } \bar{\omega}, \\
& \text { - either } q_{s}=0 \text { on } \Gamma_{e N, 1} \cap \Gamma_{\text {lat }} \text { or } \Gamma_{e N, 1} \cap \Gamma_{l a t}=\emptyset \\
& \text { - either } d_{s}=0 \text { on } \Gamma_{e N, 2} \cap \Gamma_{l a t} \text { or } \Gamma_{e N, 2} \cap \Gamma_{l a t}=\emptyset \\
& -\varphi_{0} \text { is } x_{3} \text {-affine. } \\
p=3: & \text { - the closure } \overline{\delta_{\alpha}} \text { of the projection of } \Gamma_{e D, \alpha} \text { on } \omega \text { coincides with } \bar{\omega} \\
& \text { - either } q_{s}=0 \text { on } \Gamma_{e N, 1} \cap \Gamma_{\text {lat }} \text { or } \Gamma_{e N, 1} \cap \Gamma_{l a t}=\emptyset \\
& \text { - either } d_{s}=0 \text { on } \Gamma_{e N, 2} \cap \Gamma_{l a t} \text { or } \Gamma_{e N, 2} \cap \Gamma_{l a t}=\emptyset .
\end{aligned}\right.
$$

Also, with the true physical electromechanical state $s^{\varepsilon}=\left(u^{\varepsilon}, \varphi^{\varepsilon}\right)$ defined on $\Omega^{\varepsilon}$, we associate a scaled electromechanical state $s_{p}(\varepsilon)=\left(u_{p}(\varepsilon), \varphi_{p}(\varepsilon)\right)$ defined on $\Omega$ by:

$$
\widehat{u^{\varepsilon}}\left(x^{\varepsilon}\right)=\varepsilon\left(\widehat{u_{p}(\varepsilon)}\right)(x), u_{3}^{\varepsilon}\left(x^{\varepsilon}\right)=\left(u_{p}(\varepsilon)\right)_{3}(x), \varphi^{\varepsilon}\left(x^{\varepsilon}\right)=\varepsilon^{p} \varphi_{p}(\varepsilon)(x), \forall x^{\varepsilon}=\pi^{\varepsilon} x \in \bar{\Omega}^{\varepsilon}
$$

so that $s_{p}(\varepsilon)$ is the unique solution of the following mathematical problem:

$\mathcal{P}(\varepsilon, \Omega)_{p}:$ Find $s_{p}(\varepsilon) \in\left(0, \varphi_{0}\right)+V$ such that $m_{p}(\varepsilon)\left(s_{p}(\varepsilon), r\right)=L(r), \forall r \in V=H_{\Gamma_{m D}}^{1}(\Omega)^{3} \times H_{\Gamma_{e D}}^{1}(\Omega)$, equivalent to the genuine physical one, with

$$
\left\{\begin{array}{l}
m_{p}(\varepsilon)(s, r)=\int_{\Omega} M(x) k_{p}(\varepsilon, s) \cdot k_{p}(\varepsilon, r) d x, \quad k_{p}(\varepsilon, r)=k_{p}(\varepsilon,(v, \psi))=\left(e(\varepsilon, v), \nabla_{p}(\varepsilon, \psi), \nabla_{p}^{2}(\varepsilon, \psi)\right) \\
\widehat{e}(\varepsilon, v)=\widehat{e}(v), e^{\perp}(\varepsilon, v)=\varepsilon^{-1} e^{\perp}(v), e_{33}(\varepsilon, v)=\varepsilon^{-2} e_{33}(v) \\
2 e_{i j}(v)=\partial_{i} v_{j}+\partial_{j} v_{i}, \widehat{\nabla}_{p}(\varepsilon, \psi)=\varepsilon^{p-1} \widehat{\nabla} \psi, \nabla_{p}(\varepsilon, \psi)_{3}=\varepsilon^{p-2} \partial_{3} \psi \\
\widehat{\nabla}_{p}^{2}(\varepsilon, \psi)=\varepsilon^{p-1}\left(\widehat{\nabla}^{2} \psi\right), \nabla_{p}^{2^{\perp}}(\varepsilon, \psi)=\varepsilon^{p-2}\left(\nabla^{2^{\perp}} \psi\right), \nabla_{p}^{2}(\varepsilon, \psi)_{33}=\varepsilon^{p-3}\left(\nabla^{2} \psi\right)_{33} \\
L(r)=L(v, \psi)=\int_{\Omega}(f \cdot v+q \psi+d \cdot \nabla \psi) d x+\int_{\Gamma_{m N}} F \cdot v d s+\int_{\Gamma_{e N, 1}} q_{s} \psi d s+\int_{\Gamma_{e N, 2}} d_{s} \partial_{n} \psi d s
\end{array}\right.
$$

Finding the limit problems is more difficult than in the situation of first order piezoelectricity because the case $p=1$ involves a greater number of state variables: $\widetilde{s}_{1}=(v, w, \psi, \phi)$ needs to be added to the initial state variable $s=(u, \varphi)$ and we set $\mathrm{s}_{1}=\left(s, \widetilde{s}_{1}\right)$. Let:

$$
\begin{gathered}
V_{K L}=\left\{u \in H_{\Gamma_{m D}}^{1}(\Omega)^{3} ; e_{i 3}(u)=0\right\} \\
\mathcal{V}=\left\{v \in L^{2}\left(\omega, H_{m}^{1}(-1,1)\right)^{2} \times\{0\}\right\}, \mathcal{W}=\left\{w \in\{0\}^{2} \times L^{2}\left(\omega, H_{m}^{1}(-1,1)\right)\right\} \\
H_{\partial_{3}}^{1}=\left\{\varphi \in L^{2}(\Omega) ; \partial_{3} \varphi \in L^{2}(\Omega)\right\}, H_{\partial_{3}}^{2}=\left\{\psi \in H_{\partial_{3}}^{1} ; \partial_{3} \psi \in H_{\partial_{3}}^{1}\right\}, H_{\nabla^{2}}^{2}=\left\{\phi \in H_{\partial_{3}}^{2} ; \nabla^{2^{\perp}} \phi \in L^{2}(\Omega)\right\} \\
\mathcal{E}_{1}=\left\{\varphi \in H_{\Gamma_{e D}}^{2}(\Omega) ; \phi x_{3} \text {-affine function }\right\} \\
\mathcal{E}_{2}=\left\{\psi \in H_{\nabla^{2}}^{2}, \psi x_{3} \text {-affine function, } \psi=0 \text { on } \Gamma_{e D, 1} \cap \Gamma^{l a t}, \partial_{3} \psi=0 \text { on } \Gamma_{e D, 2} \cap \Gamma^{\text {lat }}\right\} \\
\mathcal{E}_{3}=\left\{\phi \in H_{\partial_{3}}^{2}, \phi=0 \text { on } \Gamma_{e D, 1}^{ \pm}, \partial_{3} \phi=0 \text { on } \Gamma_{e D, 2}^{ \pm}\right\} \\
X_{1}=H_{\Gamma_{m D}}^{1}(\Omega)^{3} \times H_{\partial_{3}}^{1} \times \mathcal{V} \times \mathcal{W} \times H_{\partial_{3}}^{2} \times H_{\nabla^{2}}^{2}, X_{2}=H_{\Gamma_{m D}}^{1}(\Omega)^{3} \times H_{\partial_{3}}^{2}, X_{3}=H_{\Gamma_{m D}}^{1}(\Omega)^{3} \times H_{\nabla^{2 \perp}}^{2} \\
V_{1}=V_{K L} \times \mathcal{E}_{1} \times \mathcal{V} \times \mathcal{W} \times \mathcal{E}_{2} \times \mathcal{E}_{3}, V_{2}=V_{K L} \times \mathcal{E}_{2}, V_{3}=V_{K L} \times \mathcal{E}_{3} .
\end{gathered}
$$

If $\mathrm{s}_{1_{0}}=\left(0, \varphi_{0}, 0,0,0\right)$ the limit problem for $p=1$ reads as 


$$
\overline{\mathcal{P}}(\Omega)_{1}: \text { Find } \overline{\mathbf{s}}_{1} \in \mathrm{s}_{1_{0}}+V_{1} \text { such that } \int_{\Omega} M(x) k_{1}\left(\overline{\mathbf{s}}_{1}\right) \cdot k_{1}\left(\mathrm{r}_{1}\right) d x=L(r), \forall \mathrm{r}_{1}=\left(r, \widetilde{r}_{1}\right) \in V_{1}
$$

where $k_{1}\left(\mathrm{r}_{1}\right) \in L^{2}(\Omega, \mathcal{H})$ is represented by $\left(\widehat{e}\left(u^{\prime}\right), e^{\perp}\left(v^{\prime}\right), e_{33}\left(w^{\prime}\right), \widehat{\nabla} \varphi^{\prime}, \partial_{3} \psi^{\prime}, \widehat{\nabla}^{2} \varphi^{\prime}, \nabla^{2^{\perp}} \psi^{\prime}, \nabla_{33}^{2} \phi^{\prime}\right)$ when $r=\left(u^{\prime}, \varphi^{\prime}\right)$ and $\widetilde{r}_{1}=\left(v^{\prime}, w^{\prime}, \psi^{\prime}, \phi^{\prime}\right)$.

If $p=2,3$ it is possible to generalize the method described in [8]. The key point of this method is to observe that some components of $k_{p}\left(\varepsilon, s_{p}(\varepsilon)\right)$ and of $M k_{p}\left(\varepsilon, s_{p}(\varepsilon)\right)$ have vanishing limits when $\varepsilon$ goes to 0 so that it suggests a suitable orthogonal decomposition of $\mathcal{H}$ in the following subspaces:

$$
\begin{array}{ll}
\mathcal{H}_{1}^{-}=\{h=(e, g, G) ; \widehat{e}=0, \widehat{g}=0, \widehat{G}=0\} & , \mathcal{H}_{1}^{0}=\left\{h=(e, g, G): e_{i 3}=0, g_{3}=0, G_{i 3}=0\right\}, \\
\mathcal{H}_{2}^{-}=\left\{h=(e, g, G) ; \widehat{e}=0, g_{i}=0, G_{\alpha i}=0\right\} & , \mathcal{H}_{3}^{-}=\left\{h=(e, g, G): \widehat{e}=0, g_{i}=0, G_{i j}=0\right\} \\
\mathcal{H}_{2}^{0}=\left\{h=(e, g, G) ; e_{i 3}=0, \widehat{g}=0, \widehat{G}=G_{33}=0\right\}, \mathcal{H}_{3}^{0}=\left\{h=(e, g, G) ; e_{i 3}=0, g_{i}=0, G_{i 3}=0\right\} & , \\
\mathcal{H}_{2}^{+}=\left\{h=(e, g, G) ; e_{i j}=0, g_{3}=0, G_{i 3}=0\right\} & , \mathcal{H}_{3}^{+}=\left\{h=(e, g, G) ; e_{i j}=0, G_{33}=0\right\}
\end{array}
$$

For a given $p \in\{2,3\}, M$ can then be decomposed in nine elements $M_{p}^{\star \diamond} \in \mathcal{L}\left(\mathcal{H}_{p}^{\diamond}, \mathcal{H}_{p}^{\star}\right)$ with $\star, \diamond \in\{-, 0,+\}$. Hypothesis $\left(\mathbf{H}_{3}\right)$ on the electromechanical coefficients implies that $M_{p}^{00}$ et $M_{p}^{--}$are positive operators on $\mathcal{H}_{p}^{0}$ and $\mathcal{H}_{p}^{-}$. Therefore, the Schur complement

$$
\widetilde{M}_{p}=M_{p}^{00}-M_{p}^{0-}\left(M_{p}^{--}\right)^{-1} M_{p}^{-0}
$$

is an element of $\mathcal{L}\left(\mathcal{H}_{p}^{0}\right)$. It is important to note that neither $M_{p}^{00}$ nor $\widetilde{M}_{p}$ are necessarily symmetric, but nevertheless

$$
\kappa\left|h_{p}^{0}\right|_{\mathcal{H}}^{2} \leqslant \widetilde{M}_{p}(x) h_{p}^{0} \cdot h_{p}^{0}, \quad \forall h_{p}^{0} \in \mathcal{H}_{p}^{0}, \quad \text { a.e. } x \in \Omega .
$$

This is implied by the coercivity of $M\left(\right.$ see $\left.\left(\mathbf{H}_{3}\right)\right)$ and by the fundamental relation:

$$
(M h)_{p}^{-}=h_{p}^{+}=0 \Rightarrow \widetilde{M}_{p} h_{p}^{0}=(M h)_{p}^{0} \text { and } \widetilde{M}_{p} h_{p}^{0} \cdot h_{p}^{0}=M h \cdot h .
$$

The key point of the asymptotic study is to show that if $\bar{k}_{p}$ is the limit (in a suitable topology) of $k_{p}\left(\varepsilon, s_{p}(\varepsilon)\right)$, then $\left(M \bar{k}_{p}\right)_{p}^{-}=\left(\bar{k}_{p}\right)_{p}^{+}=0$ which exhibits $\widetilde{M}_{p}$ as the operator governing the two limit problems for $p=2,3$ :

$$
\overline{\mathcal{P}}(\Omega)_{p}: \text { Find } \bar{s}_{p} \in\left(0, \varphi_{0}\right)+V_{p} \text { such that } \int_{\Omega} \widetilde{M}_{p}(x) k\left(\bar{s}_{p}\right)_{p}^{0} \cdot k(r)_{p}^{0} d x=L(r), \forall r \in V_{p},
$$

where $k(r)$ stands for $(e(v), \nabla \psi)$, while $k(r)_{p}^{0}$ denotes the projection of $k(r)$ on the space $\mathcal{H}_{p}^{0}$, for all $r=(v, \psi) \in V_{p}$.

Under assumptions $\left(\mathbf{H}_{1}\right)-\left(\mathbf{H}_{5}\right)$, we have the following convergence result:

Theorem 3.1 $i)$ When $\varepsilon$ tends to 0 , the family $\left(s_{1}(\varepsilon)\right)_{\varepsilon>0}$ of the unique solutions of $\mathcal{P}(\varepsilon, \Omega)_{1}$ is such that $\left(s_{1}(\varepsilon), k_{1}\left(\varepsilon, s_{1}(\varepsilon)\right)\right.$ converges strongly in $H_{\Gamma_{m D}}^{1}(\Omega)^{3} \times H_{\partial_{3}}^{1} \times L^{2}(\Omega, \mathcal{H})$ to $\left(\bar{s}_{1}, k_{1}\left(\overline{\mathrm{s}}_{1}\right)\right)$, where $\overline{\mathrm{s}}_{1}=\left(\bar{s}_{1}, \widetilde{\bar{s}}_{1}\right)$ is the unique solution of $\overline{\mathcal{P}}(\Omega)_{1}$.

ii) For $p=2,3$ and when $\varepsilon$ tends to 0 the family $\left(s_{p}(\varepsilon)\right)_{\varepsilon>0}$ of the unique solutions of $\mathcal{P}(\varepsilon, \Omega)_{p}$ converges strongly in $X_{p}$ to the unique solution $\bar{s}_{p}$ of $\overline{\mathcal{P}}(\Omega)_{p}$.

SKETCH OF PROOF . - As usual, $C$ denotes various constants which can differ from one line to another. Taking into account Korn and traces inequalities in $H^{1}(\Omega)^{3}$ for the displacements and Poincaré-like and traces inequalities in $H_{\partial_{3}}^{1}(\Omega)$ for the electric potential and its derivatives, $\left(\mathbf{H}_{4}\right)-\left(\mathbf{H}_{5}\right)$ suffice to 
show that $\left|k_{p}\left(\varepsilon,\left(0, \varphi_{0}\right)\right)\right|_{L^{2}(\Omega, \mathcal{H})} \leq C$ and $|L(r)| \leq C\left|k_{p}(\varepsilon, r)\right|_{L^{2}(\Omega, \mathcal{H})}$. Assumption $\left(\mathbf{H}_{3}\right)$ then implies $\left|k_{p}\left(\varepsilon, s_{p}(\varepsilon)\right)\right|_{L^{2}(\Omega, \mathcal{H})} \leq C$. Thus there exist a non-relabeled subsequence and $\left(\bar{s}_{p}, \bar{k}_{p}\right)$ in $V_{p} \times L^{2}(\Omega, \mathcal{H})$ such that

$$
\left(s_{p}(\varepsilon), k_{p}(\varepsilon)\right) \rightarrow\left(\bar{s}_{p}, \bar{k}_{p}\right) \text { in } X_{p} \times L^{2}(\Omega, \mathcal{H}), \quad k\left(\bar{s}_{p}\right)=\left(\bar{k}_{p}\right)_{p}^{0}, \quad\left(\bar{k}_{p}\right)_{p}^{+}=0 .
$$

For $p \geq 2$, using suitable test functions in $\mathcal{P}(\varepsilon, \Omega)_{p}$ as in [8], we get $\left(M \bar{k}_{p}\right)_{p}^{-}=0$. Taking test functions such that $k_{p}(\varepsilon, r)=k(r)_{p}^{0}$ we conclude that $\bar{s}_{p}$ is the unique solution of $\overline{\mathcal{P}}(\Omega)_{p}$ so that the whole sequence $s_{p}(\varepsilon)$ converges. To get the strong convergence it suffices to choose $h=k_{p}\left(\varepsilon, s_{p}(\varepsilon)\right)-k_{p}$ in $\left(\mathbf{H}_{3}\right)$, integrate in $\Omega$ and go to the limit in the right hand side of the inequality obtained through (13) - (14).

If $p=1$ the test function methods does not make it possible to show that $\left(M \bar{k}_{1}\right)_{1}^{-}=0$. We therefore proceed as in [12] and [9] by introducing the additional state variables $v_{1}(\varepsilon)=\left(\widehat{u}_{1}(\varepsilon) / \varepsilon, 0\right), w_{1}(\varepsilon)=$ $\left(0,0, u_{1_{3}}(\varepsilon) / \varepsilon^{2}\right), \psi_{1}(\varepsilon)=\varphi(\varepsilon) / \varepsilon$ and $\phi_{1}(\varepsilon)=\varphi_{1}(\varepsilon) / \varepsilon^{2}$ so that $k_{1}\left(\mathrm{~s}_{1}(\varepsilon)\right)=k_{1}\left(\varepsilon, s_{1}(\varepsilon)\right)$. Moreover, for all $\mathrm{r}_{1} \in V_{1}$ there exists $r(\varepsilon)$ in $V$ such that $k_{1}(\varepsilon, r(\varepsilon))$ converges strongly in $L^{2}(\Omega, \mathcal{H})$ toward $k_{1}\left(\mathrm{r}_{1}\right)$ and $L(r(\varepsilon))$ tends to $L(r)$. Hence, we deduce the weak convergence in $X_{1}$ of $\mathrm{s}_{1}(\varepsilon)$ to the unique (see $\left.\left(\mathbf{H}_{3}\right)\right)$ solution $\overline{\mathbf{s}}_{1}$ of $\overline{\mathcal{P}}(\Omega)_{1}$. The strong convergence is proved as for $p \geq 2$.

\section{Some properties of $\widetilde{M}_{p}$}

It is interesting to give some properties of the operators $\widetilde{M}_{p}$ which supply the constitutive relations of the electromechanical plate with electric field gradient. We recall that these operators are defined for $p=2,3$. In the case $p=2$, the limit model involves a coupling between the displacement field, the electric field and the electric field gradient while in the case $p=3$, the coupling involve the displacement field and the electric field gradient only (see the definitions of the spaces $V_{2}$ and $V_{3}$ given supra). Because of the explicit expression (11) of $\widetilde{M}_{p}$ as a Schur complement, it is possible to show that

$$
\widetilde{M}_{2}=\left(\begin{array}{ccc}
\widetilde{a}_{2} & -\widetilde{b}_{2} & -\widetilde{\alpha}_{2} \\
\widetilde{b}_{2}^{T} & \widetilde{c}_{2} & \widetilde{\beta}_{2} \\
\widetilde{\alpha}_{2}^{T} & \widetilde{\beta}_{2}^{T} & \widetilde{\gamma}_{2}
\end{array}\right), \widetilde{M}_{3}=\left(\begin{array}{cc}
\widetilde{a}_{3} & -\widetilde{\alpha}_{3} \\
\widetilde{\alpha}_{3}^{T} & \widetilde{\gamma}_{3}
\end{array}\right),
$$

where $\left(\widetilde{a}_{2}, \widetilde{b}_{2}, \widetilde{c}_{2}, \widetilde{\alpha}_{2}, \widetilde{\beta}_{2}, \widetilde{\gamma}_{2}\right) \in \mathcal{L}\left(S^{2}\right) \times \mathcal{L}\left(\mathbb{R}, S^{2}\right) \times \mathcal{L}(\mathbb{R}) \times \mathcal{L}\left(\mathbb{R}^{2}, S^{2}\right) \times \mathcal{L}\left(\mathbb{R}^{2}, \mathbb{R}\right) \times \mathcal{L}\left(\mathbb{R}^{2}\right)$ and $\left(\widetilde{a}_{3}, \widetilde{\alpha}_{3}, \widetilde{\gamma}_{3}\right) \in$ $\mathcal{L}\left(S^{2}\right) \times \mathcal{L}\left(\mathbb{R}, S^{2}\right) \times \mathcal{L}(\mathbb{R})$. Considering the influence of crystallographic classes, it can be shown that in the case of a polarization normal to the plate we have the following properties:

- $\widetilde{a}_{3}$ involves mechanical terms only,

- $\widetilde{\alpha}_{3}$ never vanishes,

- $\widetilde{b}_{2}$ vanishes for the crystalline classes $m, 32,422, \overline{6}, 622$ and $\overline{6} m 2$, as in first order piezoelectricity (see [8]),

- $\widetilde{\alpha}_{2}$ always vanishes except for the classes 3,32 and $3 m$,

- $\widetilde{\beta}_{2}$ always vanishes except for the class $m$,

- when $p=2$, there is a complete electromechanical decoupling $\left(\widetilde{b}_{2}=\widetilde{\alpha}_{2}=\widetilde{\beta}_{2}=0\right)$ for the classes 422 , $\overline{6}, 622$ and $\overline{6} m 2$, nevertheless the operators $\widetilde{a}_{2}, \widetilde{c}_{2}$ and $\widetilde{\gamma}_{2}$ involve a mixture of elastic, dielectric and coupling coefficients. In these cases, $\widetilde{M}_{2}^{\varepsilon}$ is symmetric which involves a quadratic convex energy. For plates made of these piezoelectric monocrystals, the first and second order coupling effects disappear at the structural level! 


\section{References}

[1] R. D. Mindlin, 1968. Polarization gradient in elastic dielectrics. Int. J. Solids Struct. 4, 637-642, 1968.

[2] W. Voigt, 1910. Lehrbuch der Kristallphysik. B. G. Teubner, Leipzig.

[3] R. A. Toupin, 1956. The elastic dielectric. J. Rational Mech. Anal. 5, 849-915.

[4] C. A. Mead, 1962. Electron transport mechanism in thin insulating films. Phys. Rev. 128, 20882095.

[5] J. P. Nowacki, 2010. Static and dynamic coupled fields in bodies with piezoeffects or polarization gradient. Lecture notes in applied and computational mechanics, vol.26. Springer.

[6] J. Yang, 2010. Special topics in the theory of piezoelectricity. Springer.

[7] J. Yang, H. G. Zhou \& J. Y. Li, 2006. Electric field gradient effects in anti-plane circular inclusion in polarized ceramics. Proc. R. Soc. A 462, 3511-3522.

[8] T. Weller, C. Licht, 2010. Asymptotic modeling of thin piezoelectric plates. Ann. Solid Struct. Mech. 1, 173-188.

[9] T. Weller, C. Licht, 2008. Asymptotic modeling of linearly piezoelectric slender rods. C. R. Mecanique 336, 572-577.

[10] H. Brézis, 2011. Functional Analysis, Sobolev Spaces and Partial Differential Equations. Springer.

[11] P. G. Ciarlet, 1997. Mathematical Elasticity, vol. II. North Holland.

[12] F. Murat, A. Sili, 2000. Effets non locaux dans le passage 3d-1d en élasticité linéarisée anisotrope hétérogène. C. R. Acad. Sci. Paris, Série I 330, 745-750. 